\title{
Globe
}

Revue internationale d'études québécoises

\section{Étranger et territorialité}

\section{Naïm Kattan}

Volume 10, numéro 1, 2007

Étranger et territorialité

URI : https://id.erudit.org/iderudit/1000077ar

DOI : https://doi.org/10.7202/1000077ar

Aller au sommaire du numéro

Éditeur(s)

Globe, Revue internationale d'études québécoises

ISSN

1481-5869 (imprimé)

1923-8231 (numérique)

Découvrir la revue

Citer cet article

Kattan, N. (2007). Étranger et territorialité. Globe, 10(1), 31-36.

https://doi.org/10.7202/1000077ar d'utilisation que vous pouvez consulter en ligne.

https://apropos.erudit.org/fr/usagers/politique-dutilisation/ 


\title{
AVANT-PROPOS. ÉTRANGER ET TERRITORIALITÉ
}

\author{
NAÏM KATTAN \\ Ecrivain \\ Université du Québec à Montréal
}

Il fut un temps où le bannissement était un châtiment quasi équivalent à la prison. Le territoire était intrinsèque à la vie en société et encore davantage en famille. Au cours des siècles de grande migration, des Européens ont envahi, conquis et, dans les cas des Amériques et de 'Australie, peuplé des territoires éloignés de leurs lieux de naissance. Ils se sont imposés non comme étrangers mais comme découvreurs, porteurs de civilisations aptes à rehausser tous les groupes humains au niveau des Européens, les détenteurs de valeurs supérieures. Ainsi, utopies ou prétentions fallacieuses ont permis à l'étranger de s'imposer comme maître et propriétaire de territoires envahis.

Aujourd'hui, on peut difficilement faire valoir les avantages et les bienfaits du colonialisme auprès des peuples qui s'en considèrent comme les victimes. On assiste à un phénomène nouveau. Ce ne sont plus les Occidentaux porteurs de cultures et de valeurs qui s'installent dans un ailleurs inconnu afin de le réhabiliter, sinon de le faire naître à la civilisation, mais des peuples autrefois dominés qui cherchent pacifiquement, individu par individu, à faire leur entrée dans les sanctuaires de l'Occident.

Autrefois fermés aux Asiatiques, les États-Unis, le Canada et l'Australie ont fini par céder à la pression, modifié leurs lois d'immigration et éliminé les discriminations édictées en toute bonne conscience contre l'entrée des étrangers et, particulièrement, des Asiatiques. De Vancouver à Montréal et de San Francisco à New York, les quartiers chinois, autrefois des marginalités de misère et d'exotisme, sont aujourd'hui intégrés, sinon accueillis comme expressions admissibles, voire salutaires de la différence. 
Celle-ci est devenue un composite d'un Occident soucieux de faire prévaloir un comportement d'ouverture dont l'emblème est la liberté d'expression de la diversité des cultures. On assiste à des phénomènes et des manifestations qui eurent été incroyables, il y a deux ou trois générations: un quartier turc à Berlin, des commerçants iraniens à Francfort, des vendeurs de rue africains et maghrébins à Rome, Milan et Bari. Cette présence est encore plus significative au sein des cultures et des langues d'accueil. Un Marocain, installé à Amsterdam écrit des poèmes en néerlandais, un Irakien, à Turin écrit un roman en italien, un Turc se fait connaître comme écrivain suédois. Un nombre quasi phénoménal d'Africains et d'Asiatiques sont présents dans la littérature anglo-saxonne. $D^{\prime}$ autres, également nombreux, s'agglomèrent sous l'enseigne de la francophonie. Dans tous les cas, ces écrivains ne se considèrent pas comme porte-parole, même quand ils décrivent des groupes aux contours vagues et se contentent d'inscrire une mémoire.

On change de statut et de dénomination dès qu'on quitte un territoire pour un autre. On est étranger sans parvenir à définir ce à quoi renvoie une telle qualification. On peut remonter à la Bible et rappeler la prescription divine s'adressant aux Hébreux: vous devez respecter l'étranger parmi vous et lui appliquer les mêmes règles de justice qui prévalent parmi vous, car vous étiez étrangers en Egypte.

Et aujourd'hui qui est l'étranger? La description la plus simple, même si elle est lapidaire, serait: celui qui est différent. Cette différence est multiple. La couleur de la peau est la plus visible. On peut dire, sans être coupable de discrimination, qu'un Africain est différent d'un Asiatique comme celui-ci l'est d'un Européen. Des figures mitoyennes existent qui ne tombent pas dans le cadre de telles distinctions. Un Latino-américain, un Arabe du Proche-Orient ou d'Afrique du Nord peuvent être confondus avec des personnes géographiquement éloignées. Par ailleurs, les métissages donnent naissance à des figures physiques qu'on peut difficilement enfermer dans des traits distinctifs.

La différence ne s'arrête pas là. Pour commencer: la religion. Là encore, nous affrontons des difficultés à tracer des frontières. Un catholique mexicain peut considérer un catholique polonais comme étranger autant qu'un musulman de Syrie le ferait d'un musulman d'Indonésie. On peut recourir alors à des amalgames. Les chrétiens et les musulmans peuvent être asiatiques, américains, européens ou africains. Sont-ils culturellement des étrangers les uns par rapport aux autres? Souvent les frontières deviennent floues, quasi intangibles. On peut mettre un terme à l'interrogation en affirmant que l'étranger est celui qui vient d'ailleurs. Or, dans un monde en 
mouvement, cela comprendrait énormément de monde. Une séparation d'une telle ampleur est dangereuse, car dès qu'on cède à la tentation d'établir une hiérarchie entre autochtones et étrangers, le risque de discrimination fait irruption.

On peut renverser la situation et se demander comment l'étranger se voit lui-même et comment il se définit par rapport à l'autochtone. Les hommes et les femmes qui se déplacent et changent de territoire prennent conscience de leur état d'individu. On peut être forcé de franchir les frontières du pays natal et l'on peut choisir de le faire même si ce choix est quasi forcé. Quand il fuit les persécutions religieuses, politiques ou raciales dans son pays, l'etranger se sent le plus souvent de passage dans le pays refuge. Il croit que son séjour serait transitoire et espère qu'il soit court, éphémère. Le retour s'inscrirait au bout. Il s'agit, dans ces cas, d'un déplacement forcé qui est suivi d'exil. Celui-ci peut être de courte durée ou se prolonger pour toute une vie. L'exilé choisit de ne pas vivre pleinement dans le nouveau territoire. Il demeure en attente. Si celle-ci dure longtemps, le territoire quitté subit des transformations imposées par le passage des années. Or, dans son esprit, ce territoire reste figé. Pour lui cette immobilité est synonyme de fidélité, de loyauté à une idéologie, un idéal. Le temps condamne l'exilé à n'être nulle part. Il ne débarque jamais véritablement dans le nouveau pays et il ne revoit plus les rivages quittés. S'il revient dans son pays après une longue absence, il risque de ne plus le reconnaître. Sa famille, ses amis auront vieilli et s'ils ne sont pas morts, ils auront forcément changé.

Il existe une alternative à l'exil: la proclamation d'une nouvelle naissance. Celui qui quitte volontairement son pays pour échapper à des restrictions sociales ou à des impasses économiques accepte l'état d'étranger comme une chance, une porte ouverte à une nouvelle naissance. Dès lors qu'il s'engage dans la vie de son nouveau pays et met à son service son énergie et ses talents, il accepte son nouveau statut et peut s'attendre à un recommencement social et humain. Cela dépend grandement de l'accueil qu'il reçoit, mais il peut réussir à franchir les frontières et les obstacles par sa persistance et par un déploiement de volonté. Sa propre transformation n'est pas immédiate. Elle est longue et s'accomplit par étapes. La toute première est son acceptation de son nouveau territoire. À cause de son ignorance des règles différentes, il est souvent tenté, par manque de patience mais aussi de courage, de les rejeter et de dénigrer son nouvel environnement. Il prétendra par exemple que dans sa ville natale l'opéra et les concerts symphoniques lui étaient accessibles même s'il n'avait pas l'habitude de fréquenter les salles de 
spectacle. Chez lui, la cuisine était moins médiocre, les fruits plus succulents et les fleurs dégageaient un plus riche parfum. Cela pourrait n'être qu'une phase rapidement dépassée. Autrement l'enfermement volontaire dans un exil qui ne dit pas son nom prendrait le dessus. L'étranger, une fois son nouveau pays adopté, peut et doit exercer sa liberté de critiquer les aspects et les pratiques de son habitat qui, dans son esprit, devraient subir des réformes et des changements. Son rapport avec la langue est une étape plus difficile. S'il connait la langue du nouveau territoire, le problème est réglé. Il la parle avec un accent qu'il peut tenter de corriger. À condition d'admettre et de faire prévaloir le fait qu'on ne parle pas une langue sans avoir un accent. Celui de la majorité domine, mais n'en demeure pas moins un accent. Celui de l'étranger met en lumière sa différence sans le figer nécessairement dans un statut. C'est à lui d'insister qu'être différent ne signifie pas être étranger. L'apprentissage humble et persistant de la langue nouvelle est la reconnaissance implicite d'une nouvelle naissance. Il est possible de parvenir assez rapidement à s'approprier la langue comme instrument d'échange et de communication du quotidien. Cependant, l'acquisition d'une grande liberté d'expression est longue, ardue et parfois pénible. L'aboutissement n'est jamais totalement atteint, mais l'effort ouvre les portes à la lueur et à l'ardeur. Pour un écrivain, il s'agit d'une épreuve fondamentale. J'en ai fait l'expérience. Afin de passer de l'arabe au français comme écrivain, j'ai dû traverser le désert d'un silence qui a duré une vingtaine d'années.

Certains écrivains cèdent à la tentation de fournir constamment des démonstrations de leur maîtrise du nouvel instrument. Ils tentent, fut-ce inconsciemment, de dépasser en nuances, en subtilité, en richesse de vocabulaire ceux dont c'est la langue de naissance. Je peux citer de grandes réussites: Nabokov, Conrad. D'autres écrivains redoutent le transfert des images et des métaphores appartenant, pensent-ils, à leur langue d'origine, craignant de céder aux sollicitations faciles de l'exotisme et de ses artifices. Ils choisissent le contraire, un minimalisme qui les situent au seuil de l'absurde dans leur expression du complexe. C'est le cas de Ionesco. D'autres, de Gibran à Salah Stétié, en passant par Edmond Jabès et Georges Schéhadé, n'hésitent pas à transférer images et métaphores en évitant de tomber dans l'exotisme. Ils ne font qu'ajouter ainsi leur apport à la richesse de la langue d'accueil.

Le changement de langue met en lumière le passage de l'étranger à l'habitant permanent du nouveau territoire. La langue quittée n'est pas abandonnée et encore moins niée ou rejetée. Elle persiste, devient sousjacente à l'expression acquise. Ce qui persiste le plus fortement sont les 
images, celles de la vision initiale du réel. Ainsi, pour moi, la lune est encore et toujours masculine et le soleil, féminin, tels que l'arabe les a imprimés dans mon esprit, même si, obéissant à la grammaire française, j'écris le contraire. Je peux multiplier les exemples qui existent évidemment dans d'autres langues.

La frontière la plus difficile à franchir est celle du temps. L'arabe, langue sémite, met l'accent sur le présent même quand il s'agit de dire le passé ou le futur. Dès lors, le verbe français est une règle apprise et jamais totalement intégrée, intériorisée et par conséquent maîtrisée.

Le changement de langue est aussi, et je pourrais dire surtout, un passage d'un monde à un autre. Nombreux sont les écrivains qui, sans changer de langue, nous ont révélé directement en français ou en anglais, sans passer par la traduction, des univers superficiellement connus. Ainsi, le Maghreb vit dans les pages de Chraïbi, de Kateb Yacine, de Taher Ben Jelloun et d'Albert Memmi, l'Inde dans celles de Mulk Raj Anand, de Mohamed Ali et de Salman Rushdie. Naipaul et Glissant font vivre les Antilles.

Il importe de souligner une autre dimension de cette littérature. Létranger a le privilège de pouvoir se placer à distance, ce qui lui permet de voir à sa manière son pays d'adoption. Il en révèle des aspects et des dimensions qui sont imperceptibles ou qui échappent au regard de ceux qui y sont nés. Émile Olivier par exemple décrit un Montréal d'un nouveau venu qui cherche à se frayer son chemin dans les sentiers de l'inconnu qu'il apprivoise. Dans Lolita, Nabokov décrit une Amérique qu'il investit non comme voyageur de passage mais comme écrivain dont c'est le pays.

De courts séjours en Chine m'ont fait sentir que dans notre monde de grande circulation, de mouvement, les contours de l'identité ne sont jamais fixes. Jamais je n'ai eu conscience de ce que peut être l'état d'étranger plus que dans une salle de cirque à Shang Du. J'étais en compagnie de deux Canadiens. À l'entracte, les enfants nous pointaient du doigt en riant. Nous faisions alors partie de ce qu'on appelle ailleurs les minorités visibles. Quelques jours plus tard, assistant à une pièce de theâtre chanté, qu'on appelle en Occident l'opéra, j'interrogeais nos compagnons chinois sur les péripéties du spectacle, du grand classique Voyage en Occident. Où se situait cet occident pour le voyageur chinois? En Inde. Et moi, natif de Bagdad qui me considérait comme originaire d'Orient! La territorialité me glissait sous les pieds. Une autre question, lancinante, surgit: serai-je partout un étranger? Ou bien, dit autrement, nulle part ne serai-je un étranger? Du coup, le choix devient le mien. Je ne peux certainement pas 
nier, comme point de départ, en croyant effacer tout particularisme, ma différence. Ce serait condamner toute identité à la disparition, toute présence personnelle à l'anonymat. Une certaine technologie, dès que nous nous abstenons de toute tentative de la maîtriser, nous invite à nous dissoudre dans la massification et nous perdre dans un spectacle permanent, perpétuellement insignifiant.

Que retiendrais-je alors? Pour commencer, l'importance de dire mon nom et mon lieu d'origine. Je pourrai alors dire que mon territoire et ma langue ne sont pas ceux de ma naissance mais constituent des choix ultérieurs. Je cherche alors le lien, un fil de continuité et c'est ma mémoire qui vient à mon secours. Je ne suis pas de nulle part. Ma ville de naissance, ma famille, mes amis, mon enfance m'habitent et je les porte partout comme dimension de mon être. Si je tente d'adopter une langue et, à travers elle, une culture, je garde mon héritage et l'exprime. J'accepte la métamorphose. Ma mémoire sera ma sauvegarde contre l'anonymat. J'habite un territoire et refuse d'en être prisonnier. Aux yeux de certains, je constate que je suis un étranger. Je l'accepte à condition que je ne sois pas un étranger à mes propres yeux. Je vis une culture en mouvement que je découvre et invente jour après jour. Ma mémoire est présente et rend la nouveauté accessible et sans menace. Sans les nier, je m'efforce de traverser les frontières afin que nul territoire ne me soit étranger. 